\title{
LINFONEUMATOSIS QUISTICA INTESTINAL EN LA INFANCIA
}

\author{
Drs. LUIS GOIZUETA y LUIS MORENO
}

Cátedra de Pediatria del Prof. Anibal Ariztia. Hospital "Luis Calvo Mackenna". Santiago.

\section{INTRODUCCIÓN}

Presentamos este trabajo con el interés de discernir sobre el tema y de dar a conocer, una vez más, un cuadro frecuente $\mathrm{y}$ bien descrito en la Patología del adulto pero, olvidado y muy poco conocido en la Patología infantil.

Nuestro interés se despertó ante la sorpresa del número de casos encontrados en el curso de las necropsias del Hospital Luis Calvo Mackenna, en relación con la escasa casuística de la bibliografía Pediátrica.

La Linfoneumatosis Quística Intestinal conocida también con los nombres de "Neumatosis Intestinal", "Enfisema intestinal", "Neumatosis Quística Intestinal", "Linfoneumatosis Quística Gastro intestinal" es, por lo visto, una entidad rara en el niño. Moore ${ }^{1}$ relata 4 casos encontrados en 260 necropsias. Wainnight ${ }^{2}$, da cuenta de 6 casos en 240 necropsias. Botsford y Krakower " encuentran 5 casos en el curso de un año. Desde Montreal en el curso de 1934 a 1956 comínican 17 casos. De los doce casos de Mackenzie ${ }^{4}$, quién da un buen esquema de la historia de esta afección, 5 son tomados de la literatura. Monnet ${ }^{5}$ colecciona 65 casos de la literatura hasta 1957. Respecto a los adultos, este cuadro, es más frecuente, Bilger ${ }^{\text {B }}$ ha coleccionado 252 casos desde 1956 a 1959 .

\section{CONCEPTOS GENERALES}

La Linfoneumatosis Quística Intestinal, se caracteriza por la existencia de un número variable de quistes gaseosos en la pared del tubo gastro intestinal o en las estructuras que lo sostienen. Se encuentran, por tanto, estos quistes, en el estómago $e$ intestino $y$ pueden encontrarse en el mesenterio, en la cisterna de Pec. quet, en el conducto toráxico, en el peritoneo parietal, en la pared de la vagi. na $y$ vejiga $\tau_{-} 10$. La localización más frecuente, dentro de lo descrito, es el estó. mago y duodeno. Nosotros los hemos encontrado en el intestino delgado, ileon terminal, y en el intestino grueso.

El tamaño de los quistes varía desde milimetros a varios centímetros $y$, pueden ser escasos o muy numerosos, aislados o en grupos. Son comunmente sésiles, existiendo algunas formas multiloba. das. En el niño son casi siempre sésiles. Nunca se comunican entre sí.

Clases a tipos: La L.N.Q.I. se clasifica en forma "Infantil" y "Adulta". Leonard Paris ${ }^{8}$ substituye el término "Infantil" por "Aguda" y el término "Adulta", por "Crónica" de acuerdo a la evolución de las lesiones y no a la edad del sujeto.

Patogenia: La Patogenia de esta enfermedad es obscura. Muchas son las teorias invocadas pero ninguna de ellas es suficiente para explicar correctamente el fenómeno. Hay dos teorías que se estiman como las más aceptables y son generalmente las invocadas.

$1^{\circ}$ La teoría mecánica que sostiene que la formación de los quistes se realizaría por el paso del aire del tubo gastro intestinal a los linfáticos los que lo conducirian y lo difundirian por las estructuras vecinas. Hay un solo caso relatado en la literatura, en el que existía una comunicación demostrativa entre el estómago y el quiste aéreo. Por otra parte, muchos son los factores que disminuyen el valor de esta teoría. $\mathrm{Ha}$ sido imposible, por ejemplo, producir experimentaImente lesiones similares introduciendo aire desde el lumen intestinal, aunque existieran ulceraciones de la mucosa, así como tampoco, ha sido posible, con el mismo método, aumentar el tamaño de los quistes. Fi. nalmente se ha observado que la compo. sición de los gases de los quistes es diferente de la del lumen intestinal.

2 'La teoría bioquímica de Masson ${ }^{9}$, que supone una reacción ácida del contenido intestinal. Este contenido ácido sería absorbido por los capilares linfáticos y los gases se formarían por la acción del 
material ácido sobre los carbonatos con tenidos en la linfa. Posteriormente, estos gases se mezclarian con los de la sangre por difusión a través de las paredes capilares y pasarian después a las estructuras vecinas. Se pregunta, el por qué de la poca frecuencia de la neumatosis quística intestinal en relación con la alta frecuencia del contenido ácido gastro intestinal en muchos tipos de gastro-enteritis.

Consecuentemente hay en este fenómeno otros factores envueltos. Por ejemplo habría que mencionar, la avitaminosis y la modificación de la pared intestinal en la inflamación.

Contenido y composición de los gases: Los gases en el quiste están a tensión $y$ pueden explotar bajo la presión del dedo. De acuerdo con Masson el contenido varía de tiempo en tiempo $y$ de un quiste a otro quiste.

En las determinaciones gaseosas se encuentra el siguiente resultado:

Nitrógeno $73-90 \%$
Oxígeno $5-20 \%$
Anhídrido carbónico $0-15 \%$
Metano $0-7,66 \%$
Hidrógeno $0-0,7 \%$

El Nitrógeno y el Oxigeno, están constantemente presentes en proporción igual a la de la atmósfera. En lo que se refiere al Anhidrido carbónico, éste está en mayor proporción que en la atmósfera. El Metano y el Hidrógeno no se encuentran constantemente y cuando existen están en pequeña proporción.

Diagnóstico: El diagnóstico de la Neumatosis Quística se hace en la autopsia o en el curso de las intervenciones quirúrgicas. Sin embargo, la crepitación pue. de conseguirse por la palpación profunda, y tal vez el timpanismo por la percusión. La radiografía de abdomen, ocasionalmente puede ayudarnos mostrándonos asas intestinales con doble contorno y una imagen reticular generalizada del abdomen. Además la presencia de un neumo-peritoneo y de gas atrapado en el peritones, en la grasa peri-renal, en la pared de vejiga y de vagina, son signos que ayudań al diagnóstico. Los quistes gaseosos a veces pueden verse en los exámenes ıectoscópicos.
Una historia de perturbaciones gastrointestinales de larga evolución, especialmente diarreas, infecciones repetidas o un cuadro clínico de debilidad general, son los hallazgos clínicos usuales de la L.N.Q.I. en el niño.

Complicaciones: Las complicaciones más frecuentes, son la obstrucción intestinal y el neumo-peritoneo. Excepcionalmente puede encontrarse, ruptura del peritoneo seguida de muerte.

\section{Material Y MÉtodo}

El trabajo se realizó en el Servicio de Anatomía Patológica del Hospital "Luis Calvo Mackenna" y está compuesto por 1.055 necropsias, correspondientes a niños autopsiados, entre las $2^{1 / 2}$ y 8 hrs. después de la muerte, en el trienio 1957 a 1959 en que se encontró 20 casos de L.N.Q.I., motivo de nuestra observación. Las edades fluctuaron entre 5 dias y 9 meses. Las muestras fueron fijadas en solución de formalina al $10 \%$, y teñidos con Van Giesson o Hematoxilina Eosina. $\mathrm{El} \mathrm{pH}$ in. testinal, fué medido en papel de $\mathrm{pH}$ cuyo resultado osciló entre un $\mathrm{pH}$ de 6 y 8 .

En la encuesta y ficha clínica, se analizó los rubros siguientes: edad, sexo, peso, síntomas clínicos, evolución, examen de heces, diagnósticos clínicos, porción de intestino, otras localizaciones, caracte. res microscópicos y diagnóstico AnátomoPatológico.

\section{DISCUSIÓN Y RESULTADOS}

E1 estudio sobre 1.055 autopsias, ha revelado que la frecuencia de la Linfoneumatosis Quistica Intestinal es del orden de $1.8 \%$ ', lo que está de acuerdo con la impresión mundial ${ }^{\top}-z_{-} R_{-} t_{-}$.

TABLA No I

PRECUENCIA DE LA LINFONEUMATOSIS

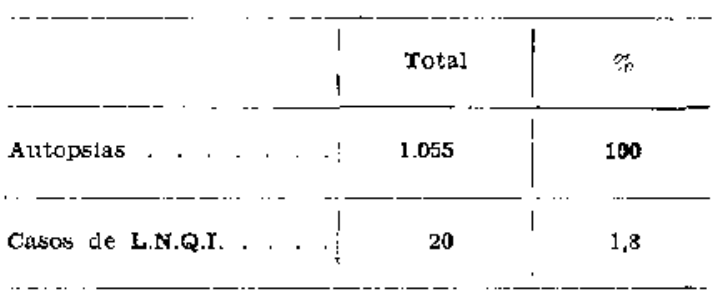


Con respecto a la distribución por edad, llama la atención en nuestro material el gran porcentaje de casos menores de 1 mes y el que todos nuestros casos son lactantes menores de un año.

No hemos encontrado diferencia significativa en cuanto al sexo.

TABLA Nơ 2

DLSTRIBUCION SECTON SEXO $Y$ EDAD

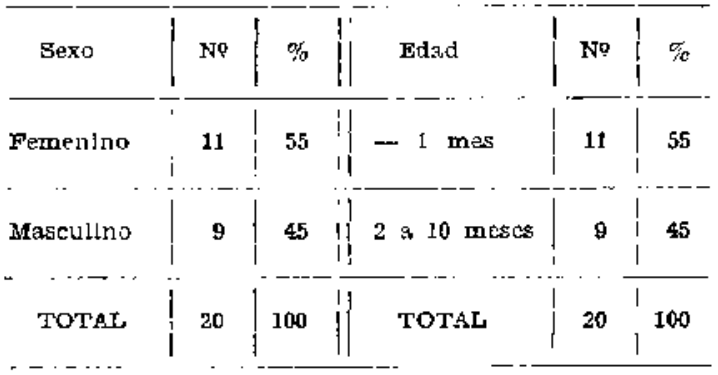

En cambio, con respecto al peso de nacimiento tomando como patrón a Prematuros con un peso menor de 2.500 grs. y normales sobre los 2.500 grs., el mayor porcentaje, está a favor de los Prematu. ros, lo que sugiere que la prematurez está relacionada con la fisiopatología de este cuadro.

TABLA N 3

DLSTRIBUCION SEGON PESO DE NACIMIIENTO

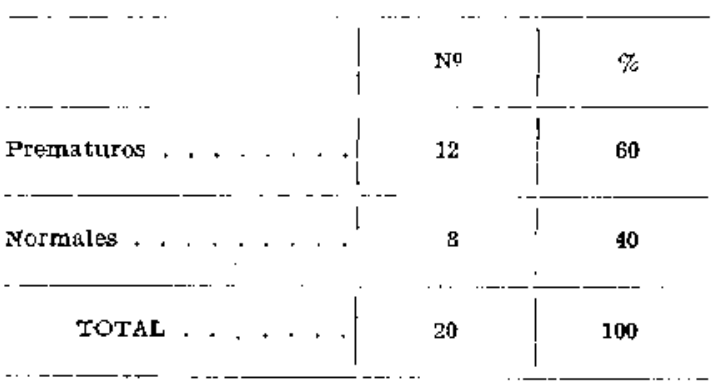

Los sintomas de comienzo más frecuentemente observados, fueron: Diarreas, Vómitos, 'Tos y Fiebre, predominando los dos primeros síntomas,
TABLA Nọ 4 SINTONAS DE COMTENZO

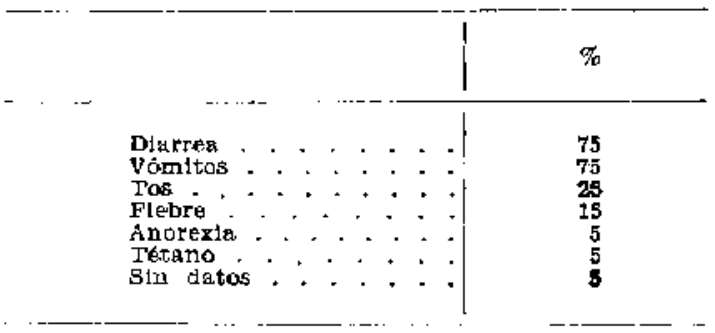

Con examen de deposiciones sólo tomamos 17 casos, ya que no se realizó en tres de ellos. De los resultados obtenidos, se deduce que el examen de deposiciones no seria una ayuda para el diagnóstico, pues. to que un alto porcentaje de ellos se encontraron negativos.

TABLA NO 5

EXAMEH DE DEPOSICIONES

(sobre 17 casos)

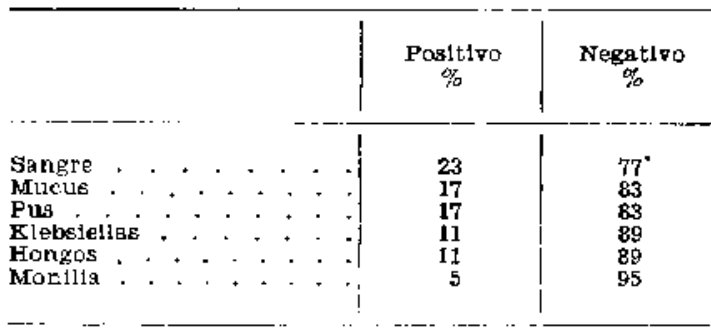

Al enfrentar el diagnóstico de ingreso con el diagnóstico Anátomo-Patológico nos damos cuenta que en ningún caso se pensó en esta posibilidad diagnóstica.

TABLA N9 6

LOCALIZACION ANATOMICA DHL QUISTE

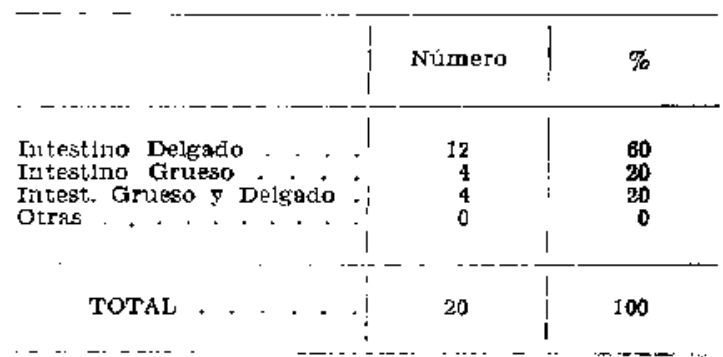


En cuanto a la localización anatómica de los quistes se aprecia que el $100^{\circ} \mathrm{c}$ de ellos están localizados en el tracto intes. tinal y en ninguno de nuestros casos hemos encontrado quistes en otro sitio como lo reportan en la literatura ${ }^{k i}$.

El mayor porcentaje correspondió al intestino delgado, y la misma frecuencia se encontró en el grueso y delgado.

TABLA NO 7

LOCALIZACION HTSTOLOGICA DEL QDLSTE

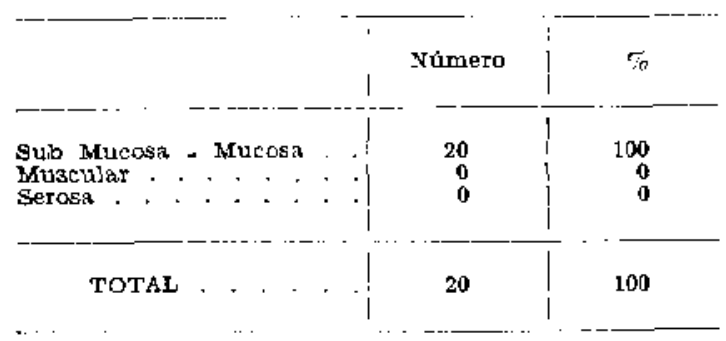

En nuestros casos el $100 \%$ de Ios quis. tes se encontraron en la sub-mucosa $y$ mucosa.

Examen microscópico: En cuanto a otros hallazgos microscópicos, el timo y el bazo presentaban picnosis y gran rúmero de linfocitos. En el bazo hay eosinófilos con localizaciones en la periferia de los folículos y congestión de la pulpa roja.

El higado mostró esteatosis periportal y en algunos casos concomitantemente hepatitis intersticial. En los pulmones revisados habia evidencia de bronconeumonía, abscesos en formación y difusa infiltración parenquimatosa.

Intestinos: En los segmentos de ileon y de ciego los quistes estaban en la submucosa, en todos los casos propulsando la mucosa y las capas musculares; las únicas regiones en que la sub-mucosa no estaba distendida era donde las fibras co. lágenas estaban firmes, abundantes y compactas. En la mayoría de los casos existieron bacterias en el lumen, sin embargo, no se aisló bacilos ni cocáceas dentro de los quistes gaseosos. Todos los casos presentaron enteritis de intensidad variable sin poder determinar en ninguno comunicación entre el lumen y los quistes. En todos los casos la mucosa estaba infiltrada por numerosos leucocitos polimorfonucleares y en el $80 \%$ por eo- sinófilos agrupados en forma difusa. En la submucosa los leucocitos eran muy numerosos, con gran predominio de eosinó filos. En las regiones donde no existian quistes no encontramos eosinófilos. En cuanto a los ganglios linfáticos mesentéricos, los encontramos hemorrágicos.

En los casos presentes de L.N.Q.I., no encontramos células gigantes, pero sí están descritas en la forma "adulta", como lo indican aquellos reportados por la $\mathrm{Li}$. teratura Médica.

\section{COMENTARIO}

Se presenta un estudio clínico-patológico de 20 casos de L.N.Q.I. en niños cuyas edades fluctuaron entre 5 días y 9 meses, así como un resumen a la literatura.

La L.N.Q.I. la hemas encontrado, con una frecuencia de $1.8 \%$. Sólo se hallaron 65 casos infantiles en la literatura. La forma infantil es del tipo Agudo $y$ se dis. tingue principalmente del tipo Adulto por la ausencia de reacción tisular en la presencia de gases. Se hace una breve exposición de la composición de los gases y de las manifestaciones clínicas prin. cipales. El estado general en todos los casos fué pobre durante un período relativamente largo de tiempo, de manera que el peso al momento de fallecer fué inferior al de su nacimiento.

La L.N.Q.I. se halló en el intestino deI. gado, íleon terminal $y$ en el intestino grueso. Las ampollas gaseosas estaban situadas principalmente en la submucosa y produjeron un abultamiento en la mucosa $y$ musculares. Se halló también que no tenían relación topográfica con las $\mathrm{uI}$ ceraciones de la mucosa presentes en algunos casos.

La Patogénesis de la L.N.Q.I. es obscura. No obstante, una hipótesis bioquímica que considera el contenido ácido intestinal $y$ otros factores, como avitaminosis e infección, se estima la más probable.

\section{FESUMEN}

Del estudio de 20 casos de Linfoneumatosis Quistica intestinal en el niño, se puede concluir Jo siguiente:

1". La Linfoneumatosis Quística resulla de la asociación de varios factores, 
donde juegan papel importante los disturbios gastro-intestinales, generalmente acompañados de procesos infecciosos, en nif̃os distróficos con avitaminosis $\mathrm{y}$ con acidificación del contenido gastro-intestinal, sin embargo la patogenia es obscura.

2. La frecuencia de acuerdo a nuestro trabajo es de $1.8 \%$ de los casos autopsiados.

3. En cuanto a la edad el $55 \%$ se presentó en el primer mes de vida, $y$ el to. tal de casos lo encontramos dentro del primer año.

$4^{4}$ Referente al sexo, no encontramos diferencias importantes.

5 Creemos que la prematurez pudiera ser un factor influyente por el alto porcentaje de neumatosis que en ellos se encuentra.

$6^{\circ}$ Comienzo de la enfermedad, en el $75 \%$ con diarreas y vómitos.

70 In la evolución en los servicios, las deposiciones se mantuvieron alteradas en el $100 \%$.

8. EI Laboratorio sería de poca ayuda, ya que en los exámenes de heces, no se apreció mayor porcentaje de alteración.

9 'El diagnóstico de este cuadro es de difjeil precisión.

$10^{\circ}$ La Iocalización, más frecuente de los quistes es el intestino, tanto delgado como grueso.

$110^{\circ}$ Las características Anátomo-patológicas, en nada difieren a los resultados de otros países, para la forma infantil.

\section{SUMMARY}

\section{LYMIPHOPNEUMATOSIS CYSTOIDES INTESTINALIS IN INFANCY}

Out of the study on 20 cases of Limphoneumatosis Cystoides intestinalis in infancy, we can extract the following points:

1) The lymphopneumatosis cystoides results from the association of various factors among which the gastro-intestinal disorders, generally acompanied by infectious processes, in dystrophic children with avitaminosis and with acidification of the gastro-intestinal contents play an important part; however, the pathogenesis is obscure.
2 i According to our study, the frequency amounts to $1,80 \%$ among the autopsied cases.

3 ) As to age, $55 \%$ presented themsel ves in the first month of life and we found all our cases within the first year of life.

4) Concerning the sex, we did not find important differences.

5) We believe that the prematurity may be an influential factor, for we found a high percentage of pneumatosis in premature infants.

6) The disease begins in $75 \%$ with diarrhea and vomiting.

7) During the evolution in the wards, the bowel evacuations remained altered in $100 \%$ of the cases.

8) The laboratory seems to be of little help, since a greater percentage of alterations was not ascertained by the examination of the feces.

9) The diagnosis of this picture is difficult to be made with precision.

10) The most frequent localization of the cysts is intestine, the small one as well as the large one.

11) The anatomo-pathological characteristics of the infantile form do not differ at all from the results of other countries.

\section{ZUSAMMENFFASSUNG}

\section{CYSTISCHE LYMPHOPNEUMATOSE DES DARMS BEIM KIND}

20 Fälle von cystische Lymphopneumatose des Darms beim Kind werden intersucht und es ergeben sich dabei folgende Schlussfolgerungen:

1) Die eystische Lymphopneumatose resultiert aus dem Zusammenwirken verschiedener Factoren, unter denen die im algemeinen mit infektiösen Processen einhergehenden Magen-Darn-Störungen bei dystrophischen Kindern mit Avitaminose und mit Ansäuerung des MagenDarm-Inhalts eine wichtige Rolle spielen; die Pathogenese ist jedoch unklar.

$2)$ Gemäss unserer Arbeit, beträgt die Häufigkeit unter den Fällen, die zur Sektion kamen, $1,8 \%$.

3 ) Was das Alter anbetrifft, so trat die Erkrankung in $55 \%$ im ersten Lebensmonat auf, und die Gesamtheit der Fälle fanden wir innerhalb des ersten Leben. sjahres. 
4) Bezüglich des Geschlechss fanden wir keine erheblichen Unterschiede.

5) Wegen des hohen Prozentsatzes der Pneumatose bei Frühgeborenen glauben wir, dass die Frühgeburt ein Faktor von Einfluss sein könnte.

6) Bei $75^{\prime:}$ begann die Krankheit mit Durchfall und Erbrechen.

7) Während des weiteren Verlaufs auf den Krankenabteilungen blieben die Darmentleerungen in $100 \% ;$ der Fälle krankhaft verïndert.

8) Das Laboratorium kann nur wenig weiterhelfen, da kein bemerkenswerter Prozentsatz von krakhaften Veränderungen in den Stühlen festzustellen war.

9) Die Diagnose dieses Krankheitsbilds ist schwer genau zu stellen.

10) Am häufigsten waren die Cysten im Darm, sowohl im Dünndarm wie im Dickdarm, lokalisiert.

11) Die pathologisch-anatomischen Charakteristika für die kindliche Form unterscheiden sich in keiner Hinsicht von den Ergebnissen in anderen Ländern.

\section{BIBLIOGRAFIA}

1.-MOORE, R. A. - Intestinal, pneumatosts, Am. J. D18. Ch1led, 38:818, 1929.

2,-WATNRIGHT, J. - Intestinal pneumatosis in Infants. Arch. Dis. Childhood. 26:164, 1951.

3.-BOSTSFORD, T. W, and KRAKOWER, c. - Pneumatasis of the intestine in infancy. J, Pediat. 13: 185.1938 .

4. -MAACRANZIE, E. P. - Pneumatosis Intestinalje. Pediatries, $7: 537$, 1951.

5. -MONNET, P. - Pneumatosis Kystique intestinale du naurrisson. Ann. paediat. 33:394, 1957.

6.-BILGBR. M. - Pneumatosis cystoldes intestinalls In chllaren, I. Fedtat, 49:445, 1956.

7. STIENNON O. A. - Preumatosis Intestinalis in the newborn. Ann. J. Dls. Chlla. $81: 651$, 1951

8.-PARIS, L. - Pneumatosis cystoldes intestinalis in Infancy. J. Pediat. 46:1, 1955.

9.-MASSÓN, P. - La Lynphopneumatose Kystlque. Ann. Anat. Path. 2:541, 1925.

10.-JDDGE, D. J, CASSIDY, J, E, and RICE, E. C Intestinal emphysema in infants. Arch. Path. 48: $206,1949$.

11,-HORMAN, I R - Kingseher Beritch weber 9 Faelle von Pneumatosis Intestine in Saugllngneter. Detitsche Gesunlh. Wis, 8:137, 1951

12.-Koss. L. G. - Abdominai gas cysts (Pneumatosis cystoldes incestinarum hominis). Arch. Path. 52: $523,1952$.

13.-ROSENBAUN, H, O - Paeumatosis cystoldes inn testinalis Report of the first case complleated by fatal rupture

14. -MARCONI, G. - Ia pnelumatosis cistica gastro Intestluale, con particolare riguerdo alla pneumatosis gastrica, nell wleera-caero. Arch. de Veaht per Alnt. pat. e ned clin. 27:603. 195?. 